\title{
Bacterial Infection and Trachoma in The Gambia: A Case-Control Study
}

\author{
Matthew J. Burton, ${ }^{1,2}$ Richard A. Adegbola, ${ }^{2}$ Fabakary Kinteb, ${ }^{3}$ Usman N. Ikumapayi ${ }^{2}$ \\ Allen Foster, ${ }^{1}$ David C. W. Mabey, ${ }^{1}$ and Robin L. Bailey ${ }^{1,2}$
}

Purpose. Trachoma is the leading infectious cause of blindness worldwide. Conjunctival scarring is initiated by recurrent Chlamydia trachomatis infection. However, disease progression to trichiasis occurs even in regions where chlamydial prevalence is currently low, which suggests that other factors, for example other bacterial infection, may also drive inflammation and scarring, particularly in the late stages of trachoma. This study was undertaken to investigate whether trachomatous trichiasis or conjunctival scarring are associated with increased prevalence of bacterial infection.

Methods. Within a case-control study design, individuals with trichiasis or conjunctival scarring (without trichiasis) were compared with normal matched control subjects. Subjects were examined for signs of trachoma. Conjunctival swab samples were collected for bacteriologic culture and C. trachomatis PCR.

REsults. Recruited for the study were 121 trichiasis casecontrol pairs and 117 conjunctival scarring case-control pairs Eyes with trichiasis were more frequently infected with bacteria $(37 \%)$ than were normal control eyes (7\%) (OR: 8.2; $P<$ 0.001; 95\% CI: 3.24-20.8). Bacterial infection was more common with increased trichiasis severity. In the conjunctival scarring case-control group, scarred eyes had slightly more bacterial infection (11\%) than did normal control eyes (6\%), although this was not significantly different (OR: $2.2 ; P=$ 0.144; 95\% CI: $0.79-6.33$ ).

Conclusions. Trichiasis is associated with increased risk of bacterial infection, and there may be a similar trend in eyes with conjunctival scarring. Bacterial infection of the conjunctiva is associated with inflammation, which may result in progressive scarring. Prospective studies are needed to determine the contribution of bacterial infection to disease progression. Bacterial infection probably also contributes to the development of corneal opacification. (Invest Ophthalmol Vis Sci. 2007; 48:4440 - 4444) DOI:10.1167/iovs.07-0315

From the ${ }^{1}$ International Centre for Eye Health, London School of Hygiene and Tropical Medicine, London, United Kingdom; ${ }^{2}$ Medical Research Council Laboratories, Fajara, The Gambia; and the ${ }^{3}$ National Eye Care Programme, Ministry of Health, Banjul, The Gambia.

Supported principally by Grant 01-030 from the International Trachoma Initiative with additional support by Grant 059134 from the Wellcome Trust/Burroughs Wellcome Fund. The funders had no part in the study design; in the collection, analysis, and interpretation of data; in the writing of the report; and in the decision to submit the paper for publication.

Submitted for publication March 14, 2007; revised May 19, 2007; accepted June 29, 2007.

Disclosure: M.J. Burton, None; R.A. Adegbola, None; F. Kinteh, None; U.N. Ikumapayi, None; A. Foster, None; D.C.W. Mabey, None; R.L. Bailey, None

The publication costs of this article were defrayed in part by page charge payment. This article must therefore be marked "advertisement" in accordance with 18 U.S.C. $\$ 1734$ solely to indicate this fact.

Corresponding author: Matthew J. Burton, International Centre for Eye Health, London School of Hygiene and Tropical Medicine, Keppel Street, London WC1E 7HT, UK; matthew.burton@1shtm.ac.uk.
B linding trachoma is a significant public health problem in some of the world's poorest regions. ${ }^{1}$ An estimated 8 million individuals are either blind or have severe visual impairment caused by trachoma. It is generally thought that repeated episodes of chronic follicular conjunctivitis (clinically active trachoma) caused by Chlamydia trachomatis infection drives a progressive inflammatory scarring process. Eventually, the eyelids turn inward (entropion), and the lashes come into contact with the eye (trichiasis), resulting in blinding corneal opacification.

Recent epidemiologic studies indicate that the scarring complications of trachoma continue to progress in regions such as The Gambia, where the prevalence of clinically active trachoma and chlamydial infection is now generally low after the introduction of control measures. ${ }^{2-7}$ The results of these studies suggest that other factors, in addition to C. trachomatis infection, may contribute to the progression of conjunctival scarring. Therefore, it is important to strengthen our understanding of the pathophysiology of trachoma, particularly in the advanced stages of the disease when blinding complications occur, to develop blindness prevention strategies.

A variety of factors may be involved in both the progression of conjunctival scarring and the development of corneal blindness. ${ }^{7}$ In addition to chlamydial infection these could include bacterial infection, ocular surface dryness, and a poorly regulated chronic inflammatory response. Recently, several studies from The Gambia have shown that bacteria can frequently be cultured from conjunctival swab samples in individuals with trichiasis. ${ }^{6-9}$ However, it is uncertain whether this represents a true increase in the risk of bacterial infection in eyes with trichiasis. It is also unknown whether people with trachomatous conjunctival scarring (without trichiasis) are at increased risk of bacterial infection. As a first step in investigating the possible contribution of bacterial infection to blinding trachoma it is necessary to determine whether individuals with trachomatous scarring or trichiasis have a significantly increased risk of bacterial conjunctival infection. Therefore, we conducted a case-control study in which individuals with trichiasis or conjunctival scarring alone were compared with normal control subjects.

\section{Methods}

\section{Ethical Permission}

This study was approved by the Gambian Government/Medical Research Council Joint Ethics Committee and was conducted in accordance with the tenets of the Declaration of Helsinki. The nature of the study was explained to participants, and their consent was received before clinical assessment.

\section{Study Participants}

In this investigation, two case-control studies were conducted: one in 2002 and the other in 2003. In the first subjects with trachomatous trichiasis (TT) presenting for surgery were compared with normal control subjects. In the second study, subjects with trachomatous conjunctival scarring (TS) without trichiasis were compared with nor- 
mal control subjects. In both studies, the control subjects were matched to cases for village of residence, sex, ethnicity, and age (within 10 years). In a few locations, it was not possible to find control subjects who met all the criteria. Under these circumstances, we recruited the person who most closely matched the criteria. One control was recruited for each case.

Trichiasis patients and their control subjects were recruited consecutively during the course of a systematic village by village trichiasis surgery program in Western Division, The Gambia. Patients with trichiasis requiring surgery were identified from the Gambian National Eye Care Programme (NECP) trachoma database, community ophthalmic nurse records, and village level screening. Normal control subjects were recruited by asking for a person of the same or neighboring household.

Individuals with trachomatous conjunctival scarring (without trichiasis) and their control subjects were recruited from 18 villages in the Upper Saloum District. The resident population of these villages was screened ( $90 \%$ coverage) for signs of trachoma during the course of other studies. Those in whom TS was diagnosed were invited to participate in this additional study.

\section{Clinical Assessment}

Subjects were assessed by an ophthalmologist or ophthalmic nurse experienced in the examination of patients with trachoma using $2.5 \times$ binocular loupes and a torch. Clinical signs were graded using the World Health Organization (WHO) trachoma grading system. ${ }^{10}$ Conjunctival inflammation was considered significant if there were prominent papillae and haziness of the tarsal blood vessels (P2 or P3). Lashes touching the cornea and other parts of the eye in primary position were counted separately. Evidence of epilation was noted: broken/ regrowing lashes or sections of eyelid denuded of lashes. If evidence of epilation was found, the extent of the epilation was recorded as less than or more than half the length of the eyelid.

Bacteriologic samples were collected from the left eye of subjects with TS and all the control subjects. For subjects with TT, the eye with trichiasis was sampled. In subjects with bilateral TT, the eye with more lashes touching the globe was selected. The conjunctiva was anesthetized with preservative-free proxymetacaine $0.5 \%$ eye drops (Minims; Chauvin Pharmaceuticals, Romford, UK). An inferior fornix swab sample was collected and immediately placed into a sterile tube containing STGG (skimmed milk-tryptone-glycerol-glucose) broth for bacterial culture. A second swab was collected for C. trachomatis PCR from the upper tarsal conjunctival surface. Both sample types were kept on ice before transfer to $-70^{\circ} \mathrm{C}$ or $-20^{\circ} \mathrm{C}$ freezers, respectively, later the same day. Individuals with trichiasis were offered surgery the same day under the auspices of the NECP. Clinically significant conjunctival infection was treated with a course of topical antibiotic.

\section{Tests for Infection}

Bacteriologic samples were plated on blood agar (aerobic and anaerobic), McConkey agar (aerobic), gentamicin blood agar, and bacitracin chocolate agar. The gentamicin blood agar and the bacitracin chocolate agar were placed in a $5 \%$ carbon dioxide incubator. All plates were incubated at $37^{\circ} \mathrm{C}$ for 48 hours, and isolated organisms were identified using standard bacteriologic techniques. Staphylococcus epidermidis and Bacillus were excluded from the analysis, as they are considered commensal rather than pathogenic at this site. The second swab sample was tested for $C$. trachomatis DNA with a PCR-based assay (Amplicor CT/NG Test; Roche), used with previously described modifications. ${ }^{11}$

\section{Analysis}

Data were analyzed in Stata 9 (StataCorp, LP, College Station, TX). This study used a matched case-control design. Therefore, conditional logistic regression was used to examine the relationship between disease state and other variables.

\section{Results}

\section{Trichiasis and Bacterial Infection}

A total of 121 trichiasis (TT) case-control pairs were recruited. The individuals with TT and control subjects had comparable demographic profiles, although, due to the limited choice of control subjects in some locations, the control subjects tended to be slightly younger than the case subjects $(P=0.005)$, and in several instances a control subject of the same sex was not available $(P=0.02$; Table 1$)$. Subjects with trichiasis had an average of 12.2 (95\% CI: 10.3-14.0) lashes touching the study eye before surgery (interquartile range: 4-16; total Range: 0 [epilating]-46). There were 72 individuals with bilateral trichiasis. In these cases the average number of lashes touching the eye not included in the study was 9.8 (95\% CI: 7.5-12.5) which was not significantly different from the study eyes (paired two sided $t$-test: $P=0.13$ ). Conjunctival inflammation was observed in many subjects with trichiasis (46\%), but in none of the control subjects.

Bacterial pathogens were more frequently isolated from conjunctival swab samples collected from eyes with trichiasis (37.2\%) than control subjects (7.4\%; Table 2). Conditional logistic regression analysis confirmed the significance of this association (OR: $8.2 ; P<0.001 ; 95 \%$ CI: 3.24-20.8). There was a significant increasing trend in the prevalence of bacterial infection with increasing burden of trichiasis (Table 2).

Corneal opacification (CC2/CC3 or CO) was associated with greater age, severe trichiasis, conjunctival inflammation, and bacterial infection (Table 3). Conjunctival inflammation was associated with bacterial infection (OR: 3.58; $P<0.001 ; 95 \%$ CI: $1.81-6.72)$. There was a significant increase in the prevalence of bacterial infection with increasing severity of corneal opacification: CC0 13\%, CC1 35\%, CC2 40\% and CC3 81\%; test for trend (Cuzak-Wilcoxon rank-sum test) $z=6.68, P<0.001$.

TABLE 1. A Comparison of Demographic and Clinical Characteristics of Individuals with Trichiasis and Their Matched Control Subjects

\begin{tabular}{|c|c|c|}
\hline & $\begin{array}{c}\text { Trichiasis Cases } \\
\quad(n=121)\end{array}$ & $\begin{array}{l}\text { Normal } \\
\text { Controls } \\
(n=121)\end{array}$ \\
\hline \multicolumn{3}{|l|}{ Demographics } \\
\hline Females & $95(78)$ & $79(65)$ \\
\hline Mean age (IQR) & $55.7(47-65)$ & $50.7(39-60)$ \\
\hline \multicolumn{3}{|l|}{ Ethnicity } \\
\hline Mandinka & $57(47.1)$ & $58(47.9)$ \\
\hline Wolof & $1(0.8)$ & $0-$ \\
\hline Jola & $48(39.7)$ & $47(38.9)$ \\
\hline Fula & $7(5.8)$ & $9(7.4)$ \\
\hline Manjago & $7(5.8)$ & $7(5.8)$ \\
\hline Karoninka & $1(0.8)$ & $0-$ \\
\hline \multicolumn{3}{|l|}{ Corneal opacity } \\
\hline $\mathrm{CC} 0$ & $56(46.3)$ & $121(100)$ \\
\hline CC 1 & $34(28.1)$ & $0-$ \\
\hline CC 2 & $15(12.4)$ & $0-$ \\
\hline CC 3 & $16(13.2)$ & $0-$ \\
\hline \multicolumn{3}{|l|}{ Trichiasis (number of lashes) } \\
\hline 0 (no trichiasis) & -- & $121(100)$ \\
\hline 0 (epilate) & $5(4.1)$ & -- \\
\hline $1-4$ & $13(10.7)$ & -- \\
\hline $5-9$ & $47(38.8)$ & -- \\
\hline $10-19$ & 35 (28.9) & -- \\
\hline $20+$ & $21(17.4)$ & -- \\
\hline Conjunctival inflammation (P2/P3) & $56(46.3)$ & $0(0)$ \\
\hline
\end{tabular}

Except where noted, the data are the number of subjects, with the percentage of the total in parentheses. 
TABLe 2. Conjunctival Bacterial Infection by Clinical Status

\begin{tabular}{lcc}
\hline & Trichiasis Cases & $\begin{array}{c}\text { Normal } \\
\text { Controls }\end{array}$ \\
\hline $\begin{array}{l}\text { Bacterial infection } \\
\text { Bacterial infection by } \\
\text { trichiasis severity* }\end{array}$ & $45 / 121(37.2)$ & $9 / 121(7.4)$ \\
Lashes $(n)$ & & \\
0 (epilate) & $1 / 5(20)$ & -- \\
$1-4$ & $3 / 13(23)$ & - \\
$5-9$ & $15 / 47(32)$ & - \\
$10-19$ & $12 / 35(34)$ & - \\
$20+$ & $14 / 21(66)$ & - \\
\hline
\end{tabular}

Data are the number of cases/total group, with the percentage of the total in parentheses.

* Test for trend (Cuzak-Wilcoxon rank-sum test) $z=6.38 ; P<$ 0.001 .

\section{Trachomatous Conjunctival Scarring and Bacterial Infection}

A total of 117 trachomatous conjunctival scarring (TS or C2/ C3) case-control pairs were recruited. The TS cases and control subjects had comparable demographic profiles, although due to the limited choice of control subjects in some locations these tended to be slightly younger than cases $(P=0.051)$ and in several instances a control of the same sex was unavailable $(P=0.7$; Table 4$)$. Bacterial pathogens were isolated more frequently from conjunctival swab samples from eyes with TS (11.1\%) than control subjects (6.0\%); however, this difference was not statistically significant (conditional logistic regression: OR: $2.2 ; P=0.144 ; 95 \%$ CI: $0.79-6.33)$.

\section{Bacterial Pathogens}

A wide range of organisms were isolated from conjunctival swab samples in these studies (Table 5). Among trichiasis cases Streptococcus pneumoniae was the commonest organism accounting for $42 \%$ of isolates. This pattern of $S$. pneumoniae dominance was not seen in the TS cases or either of the control groups.

\section{C. trachomatis Infection}

Chlamydial PCR testing was conducted on all 121 trichiasis case-control pairs. Only one individual with trichiasis had detectable $C$. trachomatis DNA; no control subjects had detectable $C$. trachomatis. In the second study of trachomatous scarring without trichiasis it was possible to only test the first 44 case-control pairs by chlamydial PCR, all whom were negative.

\section{Discussion}

These case-control studies investigated the association between conjunctival bacterial infection and trachomatous trichiasis and scarring. Eyes with established trichiasis were signifi-

TABLE 3. Multivariable Logistic Regression Model for Visually Disabling Corneal Opacification (CC2 or CC3) among All Trichiasis Cases and Controls

\begin{tabular}{lrrr}
\hline \multicolumn{1}{c}{ Variable } & OR & $\mathbf{9 5 \%}$ CI & \multicolumn{1}{c}{$\boldsymbol{P}$} \\
\hline Age 60+ years & 3.40 & $1.28-9.07$ & 0.014 \\
Severe trichiasis (10+ lashes) & 6.82 & $2.43-19.2$ & $<0.001$ \\
Severe conjunctival inflammation (P3) & 4.78 & $1.37-16.7$ & 0.014 \\
Bacterial infection & 3.96 & $1.48-10.6$ & 0.006 \\
\hline
\end{tabular}

TABLE 4. A Comparison of Demographic Characteristics and Conjunctival Bacterial Infection in Individuals with Conjunctival Scarring and Their Matched Control Subjects

\begin{tabular}{lcc}
\hline & $\begin{array}{c}\text { Scarring Cases } \\
(\boldsymbol{n}=\mathbf{1 1 7})\end{array}$ & $\begin{array}{c}\text { Normal Controls } \\
(\boldsymbol{n}=\mathbf{1 1 7})\end{array}$ \\
\hline $\begin{array}{l}\text { Demographics } \\
\text { Females }\end{array}$ & $68(58)$ & $64(55)$ \\
Mean age (IQR) & $43.5(29-58)$ & $39.9(29-51)$ \\
Ethnicity & $6(5.1)$ & $8(6.8)$ \\
$\quad$ Mandinka & $90(76.9)$ & $88(75.2)$ \\
Wolof & $21(18.0)$ & $21(18.0)$ \\
Fula & $13 / 117(11.1)$ & $7 / 117(6.0)$ \\
Bacterial infection & & \\
\hline
\end{tabular}

Except where noted, the data are the number of subjects, with the percentage of the total in parentheses.

cantly more likely to have conjunctival bacterial infection than normal control subjects. This association strengthened with increasing disease severity, indicated by the number of lashes touching the eye. It is likely that trichiasis facilitates the introduction of bacteria into the conjunctival sac. Moreover, trichiasis may also compromise the resolution of a conjunctival bacterial infection by providing a "foreign body" surface for attachment. Eyes with tarsal conjunctival scarring without trichiasis tended to have more bacterial infection than control subjects; however, this difference was not statistically significant, possibly because of a limited sample size. It is possible that we were not able to culture all bacteria in these subjects, given the environmental and logistic constraints of working in rural Africa. However, these factors would have affected both groups equally, so would not bias any inferences that are drawn.

The central event in progressive trachomatous scarring, in common with other cicatricial conditions, is probably chronic inflammation. Conjunctival inflammation could be triggered or maintained by several factors including chlamydial and bacterial infection, ocular surface dryness, a smoldering, poorly regulated immune response and mechanical irritation from trichiasis. In this study $C$. trachomatis infection was very rarely detected. Therefore, it is unlikely to play a major role in driving disease progression in this environment at present. In contrast, bacterial infection was detected more frequently, particularly when trichiasis was present. Bacterial infection has previously been associated with conjunctival inflammation in eyes with trichiasis. ${ }^{7,9}$ However, a prospective study with frequent sampling for bacterial culture over several years is needed to determine the contribution of bacterial infection to disease progression.

Earlier studies have explored the relationship between bacterial infection and clinically active trachoma in children. ${ }^{12-17}$ It was thought that in regions where seasonal epidemics of bacterial conjunctivitis occurred bacterial infection may exacerbate the pathogenesis and transmission of trachoma. ${ }^{14}$ It was suggested that the severity of conjunctival inflammation from $C$. trachomatis infection may be increased by a coinfection with other bacteria. In addition, it was thought that person to person transmission of $C$. trachomatis may be enhanced by the presence of more copious ocular secretions. However, it is difficult to draw definite conclusions from these studies as most did not have normal control groups for comparison. In addition, direct comparison with more recent studies is complicated by the use of the older MacCallan trachoma grading system. The range of organisms cultured from these children appears to be quite similar to that found in adults with scarring complication of trachoma. The lower level of Haemopbilus 
TABLE 5. Pathological Conjunctival Bacteria Isolated in the TT and TS Case-Control Studies

\begin{tabular}{|c|c|c|c|c|}
\hline & \multicolumn{2}{|c|}{ TT Case-Control } & \multicolumn{2}{|c|}{ TS Case-Control } \\
\hline & $\begin{array}{c}\text { Case } \\
(n=45)\end{array}$ & $\begin{array}{l}\text { Control } \\
(n=9)\end{array}$ & $\begin{array}{c}\text { Case } \\
(n=13)\end{array}$ & $\begin{array}{l}\text { Control } \\
(n=7)\end{array}$ \\
\hline S. pneumoniae & $19(42)$ & $1(11)$ & $1(8)$ & \\
\hline S. viridans & & $1(11)$ & $1(8)$ & \\
\hline Streptococcus group A & & & $1(8)$ & \\
\hline Streptococcus group C & $3(7)$ & $1(11)$ & & \\
\hline Streptococcus group D & & & $1(8)$ & \\
\hline Streptococcus spp. & $8(18)$ & & $1(8)$ & $1(14)$ \\
\hline S. aureus & $5(11)$ & & $4(30)$ & $2(30)$ \\
\hline Pseudomonas aeruginosa & $1(2)$ & & $1(8)$ & $1(14)$ \\
\hline Moraxella & $3(7)$ & & & \\
\hline H. influenzae & $1(2)$ & & & \\
\hline Klebsiella & $2(5)$ & & $1(8)$ & $1(14)$ \\
\hline Neisseria spp. & $1(2)$ & & & \\
\hline Coliform spp. & $1(2)$ & $5(56)$ & $2(14)$ & $1(14)$ \\
\hline Salmonella & & $1(11)$ & & $1(14)$ \\
\hline Enterobacter sakazakii & $1(2)$ & & & \\
\hline
\end{tabular}

Data are the number of subjects, with the percentage of the total in parentheses.

influenzae found in this study (compared with earlier studies in children) may be due the very effective Hib vaccination program, which was introduced into The Gambia in 1997.18

Several animal models have been used to investigate the effect of coinfection with Chlamydia and other bacteria. ${ }^{19-21}$ These studies have given varied results. In a feline model of active trachoma using Chlamydia psittaci, coinfection with streptococci exacerbated the inflammatory disease. $^{19}$ However, in a guinea pig model also using $C$. psittaci, additional infection with Staphylococcus aureus did not alter the course of the inclusion conjunctivitis. ${ }^{20}$ The effect of secondary bacterial infection was also investigated in a monkey model of active trachoma, generated by repeated conjunctival inoculation with $C$. trachomatis. Monkeys infected with Chlamydia were no more susceptible to bacterial infection than were control monkeys, and the disease severity caused by a combined infection was similar to animals only inoculated with Chlamydia. ${ }^{21}$ In a separate series of experiments conducted on monkeys with conjunctival scarring produced by repeated exposure to $C$. trachomatis, a more severe and sustained inflammatory reaction was produced by bacterial infection, compared with control animals. $^{21}$

Corneal opacification, the cause of blindness in trachoma, may result from various insults. Trichiasis is probably the single most important factor. ${ }^{7,9}$ However, new corneal opacification has been found to develop after the successful surgical treatment of trichiasis. ${ }^{9}$ This outcome suggests that other factors such as infection (bacterial and possibly chlamydial), ocular dryness, and chronic conjunctival inflammation may also contribute. Trichiasis traumatizes the corneal epithelium increasing vulnerability to secondary bacterial infection. Moreover, the increased prevalence of bacterial pathogens infecting the conjunctiva of eyes with trichiasis probably makes infection a frequent event. ${ }^{6,9}$ In the present study although severe trichiasis $(10+$ lashes touching the eye) was found to be the strongest risk factor for corneal disease, bacterial infection was also associated with increased risk of corneal opacification.

Overall, these data suggest that bacterial infection may be more important in the pathogenesis of late-stage trachoma than previously recognized. Eyes with increasing severity of disease appear to be more susceptible to bacteria. It is likely, although not proven, that the bacterial infection promotes conjunctival inflammation, contributing to progressive scar- ring. In addition, bacterial infection is probably an important factor in the development of blinding corneal opacification in trachoma.

\section{Acknowledgments}

The authors thank the ophthalmic nurses of the Gambian NECP and the field staff from the Medical Research Council Laboratories for their hard work, often under challenging conditions.

\section{References}

1. Resnikoff S, Pascolini D, Etya'ale D, et al. Global data on visual impairment in the year 2002. Bull World Health Organ. 2004;82: 844-851.

2. Dolin PJ, Faal H, Johnson GJ, et al. Trachoma in The Gambia. BrJ Opbthalmol. 1998;82:930-933.

3. Faal H, Minassian D, Sowa S, et al. National survey of blindness and low vision in The Gambia: results. Br J Opbthalmol. 1989;73:82-87.

4. Faal H, Minassian DC, Dolin PJ, et al. Evaluation of a national eye care programme: re-survey after 10 years. Br J Ophthalmol. 2000; 84:948-951.

5. Bowman RJ, Jatta B, Cham B, et al. Natural history of trachomatous scarring in The Gambia: results of a 12-year longitudinal follow-up. Ophthalmology. 2001;108:2219-2224.

6. Bowman RJ, Faal H, Myatt M, et al. Longitudinal study of trachomatous trichiasis in the Gambia. Br J Opbthalmol. 2002;86:339-343.

7. Burton MJ, Bowman RJ, Faal H, et al. The long-term natural history of trachomatous trichiasis in The Gambia. Invest Opbthalmol Vis Sci. 2006; 47:847-852.

8. Burton MJ, Bowman RJ, Faal H, et al. Long term outcome of trichiasis surgery in The Gambia. Br J Opbthalmol. 2005;89:575-579.

9. Burton MJ, Kinteh F, Jallow O, et al. A randomised controlled trial of azithromycin following surgery for trachomatous trichiasis in The Gambia. Br J Opbthalmol. 2005;89:1282-1288.

10. Dawson CR, Jones BR, Tarizzo ML. Guide to Trachoma Control. Geneva, Switzerland: World Health Organization; 1981.

11. Burton MJ, Holland MJ, Faal N, et al. Which members of a community need antibiotics to control trachoma? - conjunctival Chlamydia trachomatis infection load in Gambian villages. Invest Opbthalmol Vis Sci. 2003;44:4215-4222.

12. Harrison RW, Julianelle LA. Studies on the infectivity of trachoma. IV. On the bacteria cultivable from trachoma and clinically similar conditions. Am J Opbthalmol. 1936;19:119-125.

13. Reinhards J, Weber A, Nizetic B, et al. Studies in the epidemiology and control of seasonal conjunctivitis and trachoma in southern Morocco. Bull World Health Organ. 1968;39:497-545. 
14. Vastine DW, Dawson CR, Daghfous T, et al. Severe endemic trachoma in Tunisia. I. Effect of topical chemotherapy on conjunctivitis and ocular bacteria. Br J Opbthalmol. 1974;58:833842.

15. Woolridge RL, Gillmore JD. Bacteriological studies on trachomatous and normal persons from three areas on Taiwan. Bull World Health Organ. 1962;26:789-795.

16. Nabli B. [Bacteriology of the trachomatous eye]. Rev Int Trach 1974;51:93-102.

17. Aouchiche M, Bonnardot R, Chibane S, et al. [Trachoma and associated flora: theoretical study and practical deductions in trachoma control in Algeria. (New health survey in the region of Guerrara)]. Rev Int Trach. 1969;46:157-167.
18. Adegbola RA, Secka O, Lahai G, et al. Elimination of Haemophilus influenzae type b (Hib) disease from The Gambia after the introduction of routine immunisation with a Hib conjugate vaccine: a prospective study. Lancet. 2005;366:144-150.

19. Darougar S, Monnickendam MA, El Sheikh H, et al. In: Hobson D, Holmes KK, eds. Nongonococcal Urethritis and Related Infections. Washington, DC: American Society for Microbiology; 1977:186-198.

20. Howcroft MJ, Okumoto M, Ostler HB, et al. Staphylococcal infection superimposed on guinea pig inclusion conjunctivitis. Can J Ophtbalmol. 1978;13:39-44.

21. Taylor HR, Kolarczyk RA, Johnson SL, et al. Effect of bacterial secondary infection in an animal model of trachoma. Infect Immun. 1984;44:614-616. 